\section{Guidance system for pheromone orientation in moths}

THE explanation proposed by Kennedy and co-workers ${ }^{1}$ of a new guidance system for pheromone orientation contains several conclusions inconsistent with previous publications: (1) the conclusion that "persistent upwind flight requires decreases as well as increases in the pheromone stimulus" conflicts with the behaviour reported for another moth Anagasta kühniella ${ }^{2}$, and the persistent upwind orientation of flying Drosophila ${ }^{3}$, walking silk moths ${ }^{4}$, cockroaches ${ }^{5}$ and grasshoppers $^{6}$ to uniform and constant odours. Possibly the sustained upwind flight could not be elicited in Adoxophyes orana because the concentration in the cloud was too low: "at least 30 times lower than the average concentration in the plume".

(2) The hypothesis that zig-zagging and casting flight is a programmed function of pheromone concentration and that "the amplitude of these cross-wind movements change(s) inversely with the strength of the pheromone stimulus" cannot be supported critically by only the anecdotal comparison of $\boldsymbol{A}$. orana flight without pheromone against that in a single pheromone concentration. Alternatively these observations might only be associated with the presence or absence of pheromone.

(3) That "zig-zagging and casting flight is not a response to loss of pheromone" is not adequately supported by noting "frequent track reversals inside the pheromone corridor". An alternative conclusion is that not all turns are elicited by the same mechanism. Reversal turns not related to a plume boundary have previously been reported in other insects ${ }^{4,5}$, but this does not imply that turns made specifically at the boundary or after a change in the pheromone concentration are not elicited as a stimulus response. For example, cockroaches turn both at the plume boundary and at irregular points within the plume?

An integrated orientation system rather than the models discussed by Kennedy and co-workers ${ }^{1}$ would be more consistent with present information. In an integrated system, several factors contribute to the control of the rate and patterns of turning, including compensatory turns made to correct deviations from the preferred wind angle, reversal turns made at the plume boundaries, stochastic turns, and turns initiated internally at a regulated frequency if a turn has not yet been elicited by one of the previous factors. In addition, the concentration of pheromone must also regulate the flight speed and the preferred wind angle.
THOMAS R. TOBIN

WILLIAM J. BELL

Department of Entomology,

University of Kansas,

Lawrence, Kansas 66045, USA

1. Kennedy, J. S., Ludlow, A. R. \& Sanders, C. J. Nature 288 , 475-477 (1980).

2. Traynier, R. M. M. Can. Ent. 100, 5-10 (1968).
3. Kellog, F. E., Frizel, D. E. \& Wright, R. H. Can. Ent. 94, 884-888 (1962).

4. Kramer, E. in Olfaction and Taste Vol. 5 (eds Denton, D \& Coghlan, J. D.) 329-335 (Academic, New York 1975)

5. Bell, W. J. \& Kramer, E. J. chem. Ecol. 6, 287-295 (1980),

6. Kennedy, J. S. \& Moorhouse, J. E. Ent. exp. appl. 12 487-503 (1969)

7. Tobin, T. R. XVI int. Cong. Ent. Kyoto, Japan (1980) Science (in the press).

KENNEDY ET AL. REPLY-The new guidance system we proposed is admittedly inconsistent with earlier publications, not least our own, and was therefore not lightly arrived at. Tobin and Bell's numbered points are discussed briefly below but reference should also be made to the full account of this work which appears elsewhere ${ }^{1}$.

(1) In the long run some inconsistencies will surely turn out to reflect real differences between cases. However, for the moment note that the only two published examples ${ }^{2,3}$ of persistent upwind flying - as distinct from walking-in uniform odour are anecdotal. Our reason for mentioning the non-persistent upwind flights in the very low concentration of our tunnel-filling pheromone cloud, was existing evidence from other moths ${ }^{4,5}$ that upwind flight is retarded by high rather than low pheromone plume concentrations. However, we also mentioned nonpersistence in our side 'corridor' pheromone cloud, and the concentration in that was higher than the average in the broken pheromone plume where upwind flight was persistent ${ }^{1}$. Thus we concluded that the non-persistence recorded was due to the continuity of stimulation in the unbroken pheromone clouds, not the concentration.

(2) The quoted remark did refer only to the presence or absence of pheromone, but it rested on the non-anecdotal ${ }^{1}$ finding that onset of the pheromone stimulus reduced the amplitude of cross-wind movements while cessation of the stimulus increased it; and, in another flying moth, changing the concentration of pheromone present has likewise been shown to produce an opposite change in amplitude 4 .

(3) We expected, but did not find, some evidence of pheromone loss inducing cross-wind reversal of the flight track by Adoxophyes orana. Of course, we cannot say it never happens, but we can say that the probability of such a reversal increases significantly on entering pheromone ${ }^{1}$; and in another moth cross-wind movements had already been shown to lengthen on leaving pheromone ${ }^{5}$.

Like Tobin and Bell, we regard this guidance system as a highly integrated one ${ }^{5,6}$ but go further in regarding the stochastic reversal turns, not as a separate class of turn, but as turns initiated internally at a frequency that is regulated stochastically by the pheromone inputs.

J. S. KENNEDY

A. R. LUDLOW

C. J. SANDERS

ARC Insect Physiology Group,

Imperial College at Silwood Park,

Ashurst Lodge, Ascot,

Berkshire SL5 7DE, UK

1. Kennedy, J. S., Ludlow, A. R. \& Sanders, C. J. Physiol Ent. (in the press)

2. Kellogg, F. E., Frizel, D. E. \& Wright, R. H. Can. Ent. 94, 884-888 (1962).

Traynier, R. M. M. Can. Ent. 100, 5-10 (1968)

4. Cardé, R. T. \& Hagaman, T. E. Envir. Ent. 8, 475-484 (1979).

5. Marsh, D., Kennedy, J. S. \& Ludlow, A. R. Physiol. Ent. 3, 221-240 (1978); 6, 225 (1981).

6. Kennedy, J. S. Physiol. Ent. 3, 91-98 (1978).

\section{Tilt of the central gas disk of the Galaxy}

BLITZ ET AL. ${ }^{1}$ have suggested that the tilt of the central galactic gas disk might be explained by a bending wave instability due to a gravitational interaction between the thin gas disk and the central stellar bulge. However, the instability theory due to Bertin and Mark $^{2}$ on which this suggestion is based, is inapplicable in the case of the central gas disk. For the theory to apply, the vertical gravitational forces on the gas disk must be due largely to its own self gravity with only a small contribution from the bulge. The frequency of the bending waves of a thin self-gravitating disk is then given by ${ }^{2,3}(2 \pi G \Sigma k)^{1 / 2}$ where $\Sigma$ is the surface mass density and $k$ is the radial wavenumber of the bending wave, yielding the group velocity used by Blitz $e t$ $a l^{1}$ to predict the scaling law for the height of the bend $(h)$ as a function of radial distance from the galactic centre $(r)$.

However, the gravitational force experienced by the gas disk between $r=$ $300 \mathrm{pc}$ and $2 \mathrm{kpc}$ is dominated by the stellar potential. The central stellar bulge is in fact significantly fiattened ${ }^{4}$ (major and minor semi-axes are 2.5 and $1.3 \mathrm{kpc}$ ), while the stellar disk component (thickness $\sim 500 \mathrm{kpc}$ ) is not completely negligible in the central region ${ }^{4}$. The magnitude of the vertical gravitational acceleration at height $h$ due to the stellar potential is therefore approximately that of an oblate spheroid with mass density $\rho_{*}$ and eccentricity close to unity ${ }^{5}$, that is, $4 \pi G \rho_{*} h$. Whilst the magnitude of the vertical acceleration due to the self gravity of the bent disk can be estimated ${ }^{2}$ to be $2 \pi G k \Sigma h$. Using $\rho_{*}=6 M_{\odot} \mathrm{pc}^{-3}$ (ref. 6) and $\Sigma=6 M_{\odot \mathrm{pc}^{-2}}$ (ref. 7) at $r=1 \mathrm{kcp}$, the ratio of the stellar field acceleration to that of the gas self gravity is $\lambda / \pi$, where $\lambda$ is the wavelength of the bending wave in parsecs. Because $\lambda \sim r$ for the tilt, the 